Letter to Editor

\section{Letter to "Medial lingual lymph node metastasis in carcinoma of the tongue"}

\section{To the Editor,}

We have red with interest the paper from Eguchi et al. [1] describing If case of metastasis in a lingual lymph node located in the medial compartment. The case presentation is detailed, and the iconography is excellent.

The reported case was properly treated with upfront surgery, and the lingual metastasis was identified and effectively addressed even if it disclosed as an intraoperative unexpected finding.

The definitive pathological analysis demonstrated a radically resected tumor showing minor vascular invasion, with a lingual mass within the T-N tract confirmed as a metastasis in a lingual lymph node of the medial compartment and not an isolated second localization of the primary tumor. Furthermore, 2 adjunctive occult neck metastases were identified in the ipsilateral neck specimen.

The Authors claimed that the absence of extranodal extension and the negative margins justified the omission of postoperative adjuvant treatment.

Being aware that retrospective evaluations are always biased by the uncovered clinical evolution, the reader should take advantage of this peculiar case to reflect on the importance of adjuvant treatment in Stage IV oral cancer, and on its indications, that are not always straightforward.

In the discussion, the authors correctly acknowledge that the presence of a lingual lymph node metastasis has to be considered a negative prognostic factor warranting aggressive treatment, but this factor was not deemed sufficient to consider postoperative treatment.

Following the 7th TNM edition [2] (the one applied in this report) the pathological staging of this tumor was pT4a because of the infiltration of the extrinsic tongue musculature, and $\mathrm{N} 2 \mathrm{~b}$ because of the 2 lymph node metastases in the neck (Stage IVA). Furthermore, additional negative elements not impacting on the PTNM were the presence of a metastasis in a lingual lymph node and the presence of minor vascular invasion.

Also following the 8th TNM edition, [3] pathological staging would have been IVA, given the $\mathrm{pN} 2 \mathrm{~b}$ nodal sta- tus. Furthermore, the authors reported a tumor thickness of $15.9 \mathrm{~mm}$ and it is most likely to assume that the depth of infiltration of this tumor exceeded $10 \mathrm{~mm}$, making it a pT3.

Adjuvant therapy should be considered for head and neck squamous cell carcinomas pathologically staged as Stage III and IV [4]. However, we agree that NCCN guidelines do not provide definitive guidance in this setting.

Following the TNM staging used by the authors, postoperative radiotherapy should have not been excluded even for a radically resected pT4a tumor, regardless of the lymph node status, especially when displaying vascular invasion. The presence of 2 neck node metastases should also be considered a significant risk factor to be taken into account when considering postoperative radiotherapy. In fact, the recent ASCO Clinical Practice Guideline [5] suggest that adjuvant neck radiotherapy should be administered to patients with oral cavity cancer and pathologic N2 or N3 disease.

The presence of 2 or more lymph node metastases was considered the cutoff lymph node burden for postoperative chemoradiation in the RTOG 9501 trial by Cooper et al. [6] However, when these findings were combined with the results of the other pivotal adjuvant EORTC 22931 trial by Bernier et al, the absence of ENE and positive margins justified to omit postoperative chemotherapy, delivering only adjuvant radiotherapy [7].

Finally, the presence of multiple ipsilateral neck metastases is a significant risk factor for the presence of occult contralateral lymph node metastasis/disease in oral cavity squamous cell carcinomas $[8,9]$.

In our opinion, the take-home message to be underlined is that, in the presence of an ipsilateral N2b neck and a further lymph node metastasis in the medial lingual compartment, postoperative radiotherapy targeting the ipsilateral and contralateral neck should be strongly considered.

In light of the contralateral neck recurrence only 6 months after primary surgery, the case reported by Eguchi et al. suggests that contralateral neck dissection should be warranted after the intraoperative discovery of a suspicious medial lingual lymph node.

In conclusion, we agree with the authors on the severity of the reported clinical condition, being the task of the multidisciplinary team to analyze all available elements, to plan a 
complete therapeutic program, even more in rare occurrences as the one herein described.

\section{Funding}

The author(s) received no financial support for the research, authorship, and/or publication of this article.

\section{Declaration of Competing Interest}

The author(s) declared no potential conflicts of interest with respect to research, authorship, and/or publication of this article.

\section{References}

[1] Eguchi K, Kawai S, Mukai M, Nagashima H, Shirakura S, Sugimoto T, et al. Medial lingual lymph node metastasis in carcinoma of the tongue. Auris Nasus Larynx 2020;47:158-62.

[2] Sobin L, Gospodarowicz MK, Wittekind C. TNM classification of malignant tumours. 7th ed. Hoboken, NJ, USA: John Wiley \& Sons; 2009.

[3] Brierley JD, Gospodarowicz MK, Wittekind C. TNM classification of malignant tumours. 8th ed. Hoboken, NJ, USA: John Wiley \& Sons; 2017.

[4] National Comprehensive Cancer Network. NCCN Clinical Practice Guidelines in Oncology (NCCN Guidelines®) Head and Neck Cancers (Version 1.2020). https://www.nccn.org/store/login/login.aspx? ReturnURL=https://www.nccn.org/professionals/physician_gls/pdf/ head-and-neck.pdf. Accessed Date: February 12, 2020.

[5] Koyfman SA, Ismaila N, Crook D, D’Cruz A, Rodriguez CP, Sher DJ, et al. Management of the neck in squamous cell carcinoma of the oral cavity and oropharynx: ASCO clinical practice guideline. J Clin Oncol Jul. 2019;37(20):1753-74.

[6] Cooper JS, Pajak TF, Forastiere AA, Jacobs J, Campbell BH, Saxman SB, et al. Postoperative concurrent radiotherapy and chemotherapy for high-risk squamous-cell carcinoma of the head and neck. N Engl J Med May 2004;350(19):1937-44.
[7] Bernier J, Cooper JS, Pajak TF, Van Glabbeke M, Bourhis J, Forastiere A, et al. Defining risk levels in locally advanced head and neck cancers: a comparative analysis of concurrent postoperative radiation plus chemotherapy trials of the EORTC (\#22931) and RTOG (\# 9501). Head Neck Oct. 2005;27(10):843-50.

[8] Kowalski LP, Bagietto R, Lara JR, Santos RL, Tagawa EK, Santos IR Factors influencing contralateral lymph node metastasis from oral carcinoma. Head Neck Mar. 1999;21(2):104-10.

[9] Kurita H, Koike T, Narikawa JN, Sakai H, Nakatsuka A, Uehara S, et al. Clinical predictors for contralateral neck lymph node metastasis from unilateral squamous cell carcinoma in the oral cavity. Oral Oncol 2004;40:898-903.

Alberto Deganello*, Vittorio Rampinelli, Tommaso Gualtieri, Alberto Paderno Unit of Otorhinolaryngology, Head and Neck Surgery, University of Brescia, ASST Spedali Civili of Brescia, Brescia, Italy

Pierluigi Bonomo

Unit of Radiation Oncology, University of Florence, Azienda Ospedaliera Universitaria Careggi, Florence, Italy

Michela Buglione di Monale e Bastia Unit of Radiation Oncology, University of Brescia, ASST Spedali Civili of Brescia, Brescia, Italy

*Corresponding author at: Unit of Otorhinolaryngology, Head and Neck Surgery, Department of Medical and Surgical Specialties, Radiological Sciences, and Public Health, University of Brescia, ASST Spedali Civili di Brescia. Brescia, 25121, Italy. E-mail address: alberto.deganello@unibs.it (A. Deganello) 